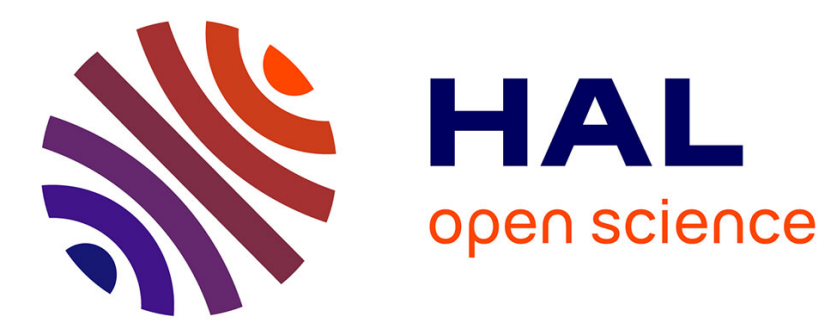

\title{
Phenomenological modelling of viscoplasticity
}

E. Krempl

\section{- To cite this version:}

E. Krempl. Phenomenological modelling of viscoplasticity. Revue de Physique Appliquée, 1988, 23

(4), pp.331-338. 10.1051/rphysap:01988002304033100 . jpa-00245778

\section{HAL Id: jpa-00245778 https://hal.science/jpa-00245778}

Submitted on 1 Jan 1988

HAL is a multi-disciplinary open access archive for the deposit and dissemination of scientific research documents, whether they are published or not. The documents may come from teaching and research institutions in France or abroad, or from public or private research centers.
L'archive ouverte pluridisciplinaire HAL, est destinée au dépôt et à la diffusion de documents scientifiques de niveau recherche, publiés ou non, émanant des établissements d'enseignement et de recherche français ou étrangers, des laboratoires publics ou privés. 


\title{
Phenomenological modelling of viscoplasticity
}

\author{
E. Krempl
}

Mechanics of Materials Laboratory, Rensselaer Polytechnic Institute, Troy, NY 12180-3590, U.S.A.

(Reçu le 26 mai 1987, révisé le 31 août 1987, accepté le 15 septembre 1987)

\begin{abstract}
Résumé.- Les bases de la modélisation phénoménologique du comportement des métaux soumis à de petites déformations sont introduites avec les expériences effectuées à l'aide de machines d'essai servocontrollées et des mesures de déformation fournies par les jauges sur les éprouvettes. L'expérimentation systématique conduit à la théorie de la viscoplasticité basée sur l'excès de contrainte, et ses propriétés sont déterminées. Avec le chargement à vitesse de déformation constante, la théorie admet les solutions asymptotiques qui sont relatives à l'écoulement plastique pur dans l'expérimentation. Une nouvelle mesure de la sensitivité de vitesse ne dépendant pas du niveau de contrainte est proposée. Les essais de relaxation, qui étaient interprétés du point de vue de la science des matériaux, sont réanalysés du point de vue phénoménologique avec des conclusions différentes.
\end{abstract}

\begin{abstract}
The essentials of phenomenological modeling of metal deformation behavior at small strain are introduced together with companion experiments which are performed with servocontrolled testing machines and strain measurement on the specimen gage length. Systematic experimentation leads to the viscoplasticity theory based on overstress and its properties are delineated. The theory admits asymptotic solutions under constant strain rate loading and they are related to fully established plastic flow in the experiment. A new measure of rate sensitivity is proposed which does not depend on the stress level. Relaxation experiments which were interpreted from a materials science viewpoint are re-analyzed from a phenomenological point of view with different conclusions.
\end{abstract}

\section{INTRODDUCTION}

In this paper a continuum mechanics, phenomenological viewpoint is adopted. The postulates of a representative volume element and of homogeneous states of stress (strain) are fundamental. It is recognized that matter consists of atoms, molecules, dislocations and other discrete particles. The aim is not to describe their motions; rather the aim is to capture the macroscopic deformation behavior of metallic materials in a mathematical model, the constitutive equation. In this case, a "smeared-out" description is adopted.

To accomplish this goal the existence of a volume element is postulated which contains a sufficient number of the microstructural, discrete elements so that its response is representative of the material under investigation. This means that the size of the volume element has to contain several grains if a polycrystalline material is considered. Within this volume the state of stress (strain) is considered to be homogeneous or uniform. A further assumption is that the material itself has homogeneity so that the properties of the representative volume element do not depend on the location within a material.

In many cases the volume element is a test specimen, frequently a cylindrical or a tubular bar, and the basic information obtained from the experiments are force-displacement pairs. One of the quantities (force or displacement) is usually prescribed as a function of time, and it is called the input; the other (displacement or force) represents the output, the answer of the material to the input. The study of input-output pairs gives information on the material and how it changes with deformation. It is the premise of a phenomenological approach that a suitable combination of input-output pairs is sufficient to obtain the essential features of the material deformation behavior in a domain of interest. Continuum mechanics provides the basic conservation laws of nature and the methods of reducing forces and displacements to proper stress and strain measures, respectively. It further generalizes the behavior and provides for predictive capabilities.

In materials sciences, macroscopic experiments similar to the ones used by the phenomenologists are used to delineate appropriate microstructural mechanisms which are then augmented by other tests, such as electron microscopy and $x$-ray analysis to name just a few (Kocks, Argon and Ashby [1]).

Since the material scientists and the mechanicians use tests on a macroscopic sample for their studies, such testing would constitute a natural starting point of much needed cooperation. The results could be interpreted by each discipline separately and then discussed jointly. Everyone 
could benefit from such a joint program.

\section{THE PHENOMENOLOGICAL APPROACH}

The deformation behavior of engineering alloys used in structural applications at homologous temperatures up to 0.6 and strain rates up to $10^{-2} 1 / \mathrm{s}$ is of interest. The strains are small and are encountered in monotonic and cyclic loadings possibly involving periods of creep or relaxation. Transients are frequent and the assumption of a steadystate behavior is rarely realistic. The task is to establish a three-dimensional constitutive equation which captures the essential features of the macroscopic deformation behavior, including Krempl [2]:

1) Rate dependence which encompasses stress and strain rate sensitivity, creep and relaxation.

2) History dependence in the sense of plasticity - sometimes called (path dependent) hardening. This term does not refer to the positive slope of a stress-strain diagram but rather describes a unique property of crystalline solids. The response to the same input can be substantially different depending on prior history. It includes such phenomena as cyclic hardening or softening. An example will be given below.

3) Recovery, the reversal of hardening by the action of diffusion.

4) Aging, the change of mechanical properties in the absence of mechanical deformation. Aging can be caused by diffusion and chemical reactions within the material, or by exchange of matter with the environment and subsequent chemical reactions.

The phenomena delineated above (except 3) are operationally defined by Kremp1 [2] and can be detected in real materials through macroscopic tests.

In this paper the emphasis will be on items 1) and 2) and it will be assumed that aging is absent. It is realized that there is another form of aging, strain aging which involves diffusion processes and chemical reactions triggered by deformation. It can lead to inverse strain rate sensitivity and other anomalous behavior. This aspect is not treated in the present context.

For the phenomenological approach, a truism of materials science (Kocks, Dawson, Follansby [3]), "The current behavior of a material is determined by its current state" is modified to read "The curreit response (output) of a material is determined by its current state and the imposed input (boundary condition)." The inclusion of boundary condition is deemed essential. In Fig.1, two annealed type 304 stainless steel bars, initially in the same state, are subjected to stress and strain control, respectively, including rate changes and unloading. (A11 test results to be quoted in this paper are obtained with strain measurement on the gage section and with servohydraulic testing machines.) It is seen that the response is different in the two cases. The effects of rate changes are much more pronounced in strain than in stress contro1; a1so, the unloading behavior is different. Significant strain accumulation is observed in stress control during unloading before the slope becomes close to the elastic value. This example demonstrates the importance of boundary conditions in testing and in assessing the properties of a materia1.

Since creep is obtained for the input "constant stress," and relaxation evolves when "constant strain" is imposed, the two phenomena are separated in a continuum approach. It is recognized that the evolution of the creep or the relaxation curve is governed by the "creep properties" of the material at a given temperature but they manifest themselves differently under different boundary conditions.

Figure 2 gives an example of history dependence for the strongly hardening type 304 stainless steel at room temperature. A virgin specimen and a specimen, which underwent completely reversed strain controlled loading to cyclic saturation followed by unloading to zero stress and strain, are subjected to the same input, strain rate cycling with two orders of magnitude difference. It is seen that the stress-strain curve of the specimen with prior cyclic loading is considerably higher than the virgin stress-strain curve, and that the stress level differences between the curve at the different rates are approximately the same for both specimens. Prior cyclic hardening has not appreciabiy changed the rate sensitivity but has significantly elevated the stress level, the manifestation of history dependence in the sense of plasticity or of (path dependent) hardening.

The results of Fig. 2 suggest that the rate sensitivity is, as first approximation, unchanged by cyclic hardening. The measure of rate sensitivity used in materials science $[1]$ is

$$
\left.\mathrm{m} \equiv \frac{\partial \ln \dot{\gamma}}{\partial \ln \sigma}\right|_{\mathrm{T}}
$$

where $\dot{\gamma}$ and $\sigma$ are the inelastic strain rate and the current stress, respectively, and where the subscript $T$ indicates that this quantity is to be determined at constant temperature.

If (1) is evaluated for the conditions of Fig.2, i.e. for the virgin stress-strain curve (subscript 1) and for the curve after prior cycling (subscript 2), with the assumption that the stress leve 1 differences are the same for 1 and 2 , then $\mathrm{m}_{1} / \mathrm{m}_{2}=\sigma_{1} / \sigma_{2}$ where $\sigma$ denotes the stress level. Since the stress levels in Fig. 2 are roughly different by a factor of two, the $m$ values differ by the same factor. This result is at variance with the result of the visual inspection that the rate sensitivity has not changed. The quantity $\mathrm{m}$, therefore, is not a sole measure of rate sensitivity but can include the effects of stress level as well.

\section{THEORY OF VISCOPLASTICITY BASED ON OVERSTRESS}

Systematic experiments on different materials form the basis of the development of the theory which can be described as experimentally based. Such tests included: type 304 stainless stee 1 (Kremp1 [4], Kujawski, Kallianpur and Kremp1 [5], Kremp1 and $\mathrm{Lu}[6]$ ); a Titanium alloy (Kujawski and Kremp1 [7]); a ferritic pressure vessel steel (Kremp1 and Kallianpur [8]); and a nickel base superalloy at $815^{\circ} \mathrm{C}$ (Kremp1, Lu and Yao [9]).

\section{The Isotropic, Cyclic Neutral Mode1}

The theory is of the "unified type" and does not use a yield surface and loading and unloading conditions. Elastic and inelastic strain rates are always present but, in regions where the deformation behavior is nearly linear (the elastic regions), the inelastic strain rate is extremely small and negligible. The "base" model is for isotropy, small strain, and represents cyclic neutral behavior. It is given by 


$$
\begin{gathered}
\dot{e}_{i j}=\dot{e}_{i j}^{e l}+\dot{e}_{i j}^{i n}=\frac{1+\nu}{E} \dot{s}_{i j}+\frac{3}{2} \frac{1}{E k[\Gamma]}\left(s_{i j}-g_{i j}^{d}\right) \\
\dot{g}_{i j}^{d}=\frac{2}{3} \psi[\Gamma] \dot{e}_{i j}-\frac{\dot{\varphi}}{b[\Gamma]}\left(g_{i j}^{d}-\frac{2}{3} E_{t} e_{i j}\right) \\
\dot{\varepsilon}_{k k}=\frac{1-2 \nu}{E} \dot{\sigma}_{k k} .
\end{gathered}
$$

In the above, a superposed dot indicates "time derivative," and square brackets following a symbol denote "function of." The symbols $\underline{\sigma}$ and $\underline{\varepsilon}$ designate the stress and the small strain tensors, respectively, with $\underline{s}$ and $\underline{e}$ the corresponding deviators, E and $\nu$ are the elastic constants, and $\underline{g}_{d}$ is the deviatoric equilibrium stress, with $\underline{s}-\bar{g}$ as the overstress. The quantity $\dot{\varphi}=\left(\frac{2}{3} \dot{\epsilon}_{i j}^{i n} \dot{\varepsilon}_{i j}^{i n}\right)^{1 / 2}$ is the rate of inelastic strain path length. The overstress invariant $\Gamma$ and the function $b[\Gamma]$ are given as

$$
\begin{gathered}
\Gamma=\left(\frac{3}{2}\left(s_{i j}-g_{i j}^{d}\right)\left(s_{i j}-g_{i j}^{d}\right)\right)^{1 / 2} \\
b[\Gamma]=\frac{A}{\psi[\Gamma]-E_{t}}
\end{gathered}
$$

The function $k[\Gamma]$ is the viscosity function, positive and decreasing with the dimension of time, and it controls the rate sensitivity. The function $\psi[\Gamma]$ is both positive and decreasing and has the dimension of stress. It controls the shape of the stress-strain diagram and is called the shape modulus function. The quantity $\mathrm{E}_{t}$ is the slope of the stress-strain curve at the highest strain of interest. It can be positive for work hardening, negative for work softening, and can be set to zero if the stress-strain diagram is horizontal. (Note that $E_{t}$ is not related to the path dependent hardening discussed previously.

When reducing the above equations to the uniaxial state of stress, Poisson's ratio is to be set equal to $1 / 2$ in the deviatoric strain quantities in Eq. (3). The properties of Eqs. (2) - (4) are evaluated by Yao and Kremp1 [10], and Kremp1, McMahon and Yao [11]. They apply the equations to predict nonproportional in-phase and out-of-phase loading of the cyclic neutral and rate insensitive 6061 T6 A1 alloy with very reasonable results.

Equations (2) - (4) contain only one state variable, the equilibrium stress, for which a growth law is given. The equilibrium stress is the stress which is obtained as all rates go to zero; the model therefore predicts that a nonzero stress can be supported at rest. (Stresses at equilibrium are called the mechanical threshold by Kocks et a1. [1, p.6]; it is thought that the equilibrium stress could be the threshold stress which evolves with deformation.) It is further evident from Eq. (2) that the inelastic strain rate is in the direction of the overstress deviator and this formulation is supported by recent experiments: Phillips [12]; Oytana, Delobelle and Mermet [13]; and McDowe11 [14]. In this paper the terms "equilibrium stress" and "overstress" are used, instead of the terms "backstress" (or "kinematic stress") and "effective stress" employed in materials science. These expressions can be considered synonyms. However, because of the fundamental assumptions of a representative volume element in which a homogeneous state of stress (strain) exists, we cannot accommodate the concept of an internal stress in a continuum analysis. (If an internal stress is assumed to exist, it must equal the applied stress when averaged over the volume element.) These aspects and the origin of the terms are discussed in detail in Kremp1 [15].

Since the theory contains only one state variable it is restricted in its modeling capabilities. It represents cyclic neutral material behavior, Yao and Kremp1 [10] and Krempl et a1. [11]; also, aging and recovery are not included in the above formulation.

The present theory is very close to the unified theories, see the recent review by Walker [16]. However, the growth law contains the total deviatoric strain and strain rate. A materials science viewpoint would suggest that such a formulation is inappropriate. The state variable is representative of microstructural changes which evolve only with inelastic deformation. While this reasoning is certainly correct, it overlooks the fact that the constitutive equation also has to model elastic ranges. It is for this reason that the total deviatoric strain rate multiplies the shape modulus function in Eq. (3); see Kremp1 et al. [11] for further discussion. The dependence on the total deviatoric strain in Eq. (3) can be eliminated by setting $\mathrm{E}_{\mathrm{t}}=0$. If this is done, then all stressstrain curves become horizontal as in many unified theories. Having $E_{t}$ different from zero permits positive and negative slopes in the plastic range which is considered an advantage for modeling.

\section{Asymptotic Solutions and Flow Stress}

Under a constant strain rate loading, Eqs. (2) and (3) admit asymptotic solutions which are mathematically valid as time approaches infinity, and which are useful within the range of small strain for appropriately chosen material constants (Yao and Kremp1 [10], Kremp1 et a1. [11] and Cernocky and Kremp1 [17]). For proportional, constant strain rate loading the asymptotic solutions are

are

$$
\left\{\dot{\sigma}_{\mathrm{e}}\right\}=\left\{\dot{\mathrm{g}}_{\mathrm{e}}^{\mathrm{d}}\right\}=\mathrm{E}_{\mathrm{t}} \dot{\varepsilon}_{\mathrm{e}}
$$

$$
\left\{\sigma_{\mathrm{e}}\right\}=\left(E-\mathrm{E}_{\mathrm{t}}\right) \mathrm{k}[\{\Gamma\}] \dot{\epsilon}_{\mathrm{e}}+\mathrm{A} \frac{\dot{\varepsilon}_{\mathrm{e}}}{\dot{\dot{\varphi}}}+\mathrm{E}_{\mathrm{t}} \epsilon_{\mathrm{e}} .
$$

Braces denote asymptotic values and the effective strain $\varepsilon_{e}=\left(2 / 3 \epsilon_{i j} \epsilon_{i j}\right)^{1 / 2}$ and the effective stress $\sigma_{e}=\left(3 / 2 s_{i j} s_{i j}\right)^{1 / 2}$. The corresponding rates are obtained simply by replacing stress or strain with its corresponding time derivative, i.e. $\dot{\sigma}_{e}=\left(3 / 2 \dot{s}_{i j} \dot{s}_{i j}\right)^{1 / 2}$. In evaluating the asymptotic effective strain and strain rate, a Poisson's ratio of $1 / 2$ is to be used in consideration that plastic flow is fully established when Eq. (8) applies.

If the first term on the right-hand side of $\mathrm{Eq}$. (8) is rewritten as

$$
\left(E-E_{t}\right) k[\{\Gamma\}] \dot{e}_{e} \equiv\{\Gamma\},
$$

the total asymptotic effective stress is composed of the rate-dependent or viscous contribution $\{\Gamma\}$, the plastic or rate-independent contribution $A \dot{\varepsilon} e_{e} / \dot{\varphi}$ and the contribution due to the plastic tangent slope $E_{t} \epsilon_{e}$. This relation is depicted in Fig. 3 .

Equation (7) clearly shows that ultimately the growth of the stress equals that of the equilibrium stress and that both are governed by the tangent modulus $E_{t}$ which can be positive, zero or negative. Therefore work hardening, no hardening, or work softening can be modeled. 
The role of the viscosity function is apparent from Eq. (9). The overstress invaitiant $\Gamma$, which might be called the effective overstress, is nonlinearly related to the strain rate via the viscosity function $k[\Gamma]$. This fact enables the modeling of the highly nonlinear strain rate sensitivity of metals and alloys; the strain race must be changed by orders of magnitude to obtain an appreciable effect on the stress leve1. Various $k[$ functions are discussed by Kremp1 [18].

The asymptotic solutions are of special importance in modeling real alloy behavior. It is seen from $\mathrm{Eq}$. (7) that in this case the tangent modulus is $E_{t}$, which physically means that plastic flow is fully established and that the "flow stress" or a steady-state flow behavior of the alloy is said to have been reached. This interpretation is adopted when the viscoplasticity theory based on overstress is applied to materials modeling. Asymptotic solution and "flow stress" are used interchangeably. If the material functions of the theory are appropriately chosen, the asymptotic solutions are rapidly attained after a change in rate. This corresponds to the conditions of Fig. 2 where the "flow stress" characteristic of the strain rate is quickly reached after a change in rate. (Note that the speed with which the "flow stress" is approached depends on the imposed boundary condition or on the type of control. In strain control the transition is rapid (hard testing machine); in stress control (soft testing machine) the transition is slow. This fact is reproduced by the model; compare Figs. 1 and 3 of Liu and Krempl [19], also Figs. 6, 7 and 10 of Kremp1 et a1. [11]. Such differences are also observed in experiments, see Fig. 1 of this paper.) The theory predicts that this "flow stress" is reached independent of the initial conditions which do not enter into the asymptotic solutions at a11. Therefore no strain rate history effect is modeled by the theory. In this context the presence of a strain rate history effect would manifest itself by the existence of two different "flow stress" curves for the same rate depending on the prior history.

In materials science a steady-state condition is postulated at sufficiently large strains characterized by constant state variables; see p. 2 of Kocks et a1. [1]. This steady-state condition most likely refers to much larger strains than are of interest here. In analogy to this postulate of materials science, we consider the regions where the asymptotic solutions are valid as regions of quasi-constant state and refer to them as regions of fully-established inelastic flow or as regions where the "flow stress" for a given stress or strain rate is reached. Since we permit an increase in the state variable $\mathrm{g}^{\mathrm{d}}$, we do not exactly match the constant state condition required by the definition of Kocks et a1. [1]. The overstress and therefore the inelastic strain rate are constant when the flow stress is reached and this fact is the justification for considering this region a region of quasi-constant state.

\section{Overstress Dependence of Inelastic Strain Rate}

In Eq. (2) the inelastic strain rate is solely a function of overstress which is akin to the effective stress concept in materials science. This dependence was first introduced by purely phenomenological, mathematical reasoning in connection with the asymptotic solutions, see Cernocky and Kremp1 [17, p.193]. In essence, the overstress dependence of the inelastic strain rate permits the modeling of the observed nonlinear relation between strain rate and the stress at any stress or strain value once the flow stress is reached. This property is considered essential. Subsequently the overstress dependence was shown to be useful in explaining relaxation and creep behavior; see Kujawski et a1. [5] and Liu and Kremp1 [19]. Kremp1 and Kaliianpur [8], and Kremp1 [18] have shown that anomalous creep behavior can be explained by the overstress dependence. It is also vital in modeling the relaxation behavior around a hysteresis loop where it is found that the relaxation or creep is much less pronounced in regions of nearly elastic slope than in regions of reduced slope. Specifically, at the same stress or strain level, creep or relaxation is much less on unloading than on loading. The overstress dependence of the inelastic strain rate reproduces these "anomalies" in a natural way; see Kremp1, Lu, Satoh and Yao [20]. Kremp1 and Kallianpur [8] have also reported experimental results which show that creep rate does not necessarily increase with creep stress; again the explanation of this fact is easy once the overstress dependence of the inelastic strain rate is introduced.

To focus the discussion, creep test results for stainless steel at $650^{\circ} \mathrm{C}$ by Ohashi, Kawai and Momose [21] are given in Fig.4. The stress leve1 of the creep test is kept constant in all tests; the first test is a conventional creep test, the others are a11 creep tests performed after prior plastic straining and subsequent unloading to the desired stress leve1 (see insert). It is seen that the accumulated creep strain is always less for the creep test performed after unloading than for that performed after loading. Moreover, as the prior plastic strain increases, the creep strain reduces significantly.

Current creep theories postulate that the creep strain rate depends on stress (Norton creep) or on stress and creep strain (strain hardening theory). They cannot explain the observations of Fig.4, rather they predict the same creep strain rate for a11 cases. If the creep strain rate is thought to depend on the overstress the results can be modeled. A growth law for the equilibrium stress is required which decreases the overstress at the beginning of each subsequent creep test. Such a growth is depicted in Fig.5. The dashed curve represents the growth of $\mathrm{g}$ at the various unloading events to the same creep stress. It is seen that $\sigma-g$ decreases as the plastic strain increases thus predicting a decreasing creep rate as observed.

\section{Extension to Cyclic Hardening/Softening}

Based on phenomenological evidence the theory has been extended to reproduce path dependent hardening applied to in-phase and out-of-phase cyclic loading of type 304 stainless steel (Krempl and Lu [6], Krempl and Lu [22], and Krempl and Yao [23]).

When cycle dependent changes are observed, the stress level reached at a certain strain changes with cycles. Following Fig. 3 the asymptotic stress is assumed to be composed of a viscous or rate-dependent contribution, plastic or rateindependent contribution, and a contribution to the slope $E_{t}$. Therefore the question must be asked which contribution to the stress changes with cycles. The answer must come from suitable experiments or from microstructural reasoning. Krempl and $\mathrm{Lu}$ [6] have shown that for 304 stainless stee1 at room temperature the changes are predominantly plastic in nature. For another material and another temperature other components may change.

With the experimental observation that the 
changes in stress level are predominantly plastic, a rate-independent growth law was formulated for the quantity A to represent cyclic hardening (see Kremp1 and Yao [23]). New measures of path length were introduced to represent the peculiar phenomena of cyclic hardening in in-phase and out-of-phase cycling. If the viscous contributions to the stress were to change, then the $k[$ ]-function has to be modified. One possibility is to make it a function of $\Gamma$ and of the inelastic strain path length.

It is not the purpose of this paper to discuss in detail the cyclic hardening phenomena. The important point is to note that the viscoplasticity theory based on overstress has only one state variable for cyclic neutral behavior. Two state variables, the equilibrium stress which can be considered a kinematic tensor, and the quantity $\mathrm{A}$ which has the same purpose as the isotropic variable in plasticity, are considered necessary to represent cycle dependent changes. A similar situation is found for the viscoplasticity theory proposed by Bena1la1 and Marquis [24].

\section{Modeling of Recovery}

At the present time the viscoplasticity theory based on overstress has not been applied to conditions where recovery is significant. In search for quantitative measures of recovery, nickel base superalloy specimens were subjected to rest periods not exceeding 33 hours after prior plastic deformation. The test temperature was $815^{\circ} \mathrm{C}$. No effect of the recovery period on the subsequent deformation behavior was found (see Krempl et a1. [11]). It was concluded that recovery was not important for the above test condition.

The aim of future work is to quantify the effect of recovery and to incorporate it into the growth laws for the equilibrium stress and for the quantity A using the standard hardening-recovery format. (The present formulation is all hardening.)

\section{DISCUSSION}

\section{Re-analysis of Published Data}

The viscoplasticity theory based on overstress was introduced and it was emphasized that it is based on phenomenological experimentation which distinguishes stress from strain control. While the theory is very close to the unified theories, it contains some unusual features such as postulating the cyclic neutral behavior for the base model. Because of these differences it is thought to be instructive if test data, which had been presented and interpreted by others, are revisited and looked at in the light of the properties of the viscoplas ticity theory based on overstress.

The test results are reproduced in Figs. 6 and 7 as the starting point of the discussion. These figures appear as Figs. 2 and 7 in Rohde, Jones and Swearengen [25] which will be referred to as "RHS," and as Figs.5A and 5B in Swearengen and Holbrook [26] which will be referred to as "SH."

Figure 6 shows the load-total strain diagram for three relaxation tests performed on pure aluminum at room temperature. The corresponding log stress versus $\log$ inelastic strain rate curves are depicted in Fig.7. (Note that there is a factor of ten discrepancy between the strain values on the abscissa of Fig. 6 and the values quoted in the caption of Fig.7.)

In RHS the relaxation data are fitted with an algebraic relation between the inelastic strain rate and the stress minus the internal stress; no evolution equations are given. When the different relaxation curves are numerically approximated, different constants are found which are given in Fig.7. Among other items the authors conclude:

1) The crossover of the reload relaxation curves is unexpected.

2) Relaxation is not a constant state process at smal1 plastic strains.

3) The best fit of the algebraic equation was obtained with setting the internal stress equal to zero.

The same data is discussed again in $\mathrm{SH}$ and the following additional conclusion is made:

4) To describe the history dependent relaxation behavior a greater number of state variables is required. (Prior discussion centers around the use of one state variable.)

When the results of Figs. 6 and 7 are analyzed with the overstress model in mind, the following points are relevant:

a) The flow stress curves are nearly horizontal and the equilibrium stress is therefore constant when the flow stress is reached.

b) During relaxation the equilibrium stress does not reduce to zero; at an inelastic strain rate of $10^{-10} \mathrm{~s}^{-1}$ the stress is nonzero. At this rate the stress is close to the equilibrium stress, see Fig. 6.

c) During the reloadings the equilibrium stress changes together with the stress. (The full equations must be integrated to obtain the stress and equilibrium stress.) At the beginning of the three relaxation tests the overstress can be quite different. As a consequence the relaxation rates are different and different relaxation curves are expected. There is no need to introduce additional state variables.

d) Since the overstress is constant when the flow stress is reached, a11 relaxation curves started from a flow stress curve obtained with the same strain rate should be identical irrespective of the strain (and stress if work hardening occurs) at which the relaxation process starts. This apparently is the experimental outcome.

This example suffices to show the difference between the phenomenological and the materiais science approaches. Further discussion on such subjects will undoubtedly improve the understanding and modeling of plasticity.

\section{A New Measure of Rate Sensitivity}

It was remarked previously that the rate sensitivity measure $\mathrm{m}$ given in Eq. (1) is sensitive to the stress level. To circumvent this problem, a new quantity $M$ was introduced by Kremp1 [18] and this quantity was entirely determined by the $k[]-$ function, and it changes with strain rate as demonstrated in Fig. 8 of that reference.

This measure $M$ can be generalized to three dimensions using the properties of the viscoplasticity theory based on overstress. It is defined as

$$
\mathrm{M} \equiv \frac{\partial \ln \dot{\epsilon}_{\mathrm{e}}}{\partial \ln \Gamma}=1-\frac{\Gamma \mathrm{dk}[\Gamma] / \mathrm{d} \Gamma}{\mathrm{k}[\Gamma]}
$$

This measure applies to monotonic loading when the 
flow stress is reached and to creep (Poisson's ratio is to be set to $1 / 2$ in the effective strain rate). For relaxation the inelastic strain rate is to be replaced by $\dot{\varepsilon}_{e}=\dot{\sigma}_{e}(1+\nu) / E$. This measure depends only on the overstress and applies in any kind of proportional loading. It is known once the $k[$ ]-function of a material has been determined.

\section{ACKNOWLEDGEMENT}

The financial support of the U.S. Department of Energy is gratefully acknowledged. S. Han provided the translation of the Abstract.

\section{RLFERENCES}

1) Kocks, U.F., Argon, A.S., and Ashby, M,F., Thermodynamics and Kinetics of Slip, Pergamon Press, Oxford, 1975.

2) Kremp1, E., "Plasticity and Variable Heredity," Arch. Mech., 33, 1981, 289-306.

3) Kocks, U.F., Dawson, P.R., and Follansby, P.S., Physical and Phenomenological Plasticity, Los Alamos Center for Materials Science, Report LA-UR-86-2672, see p.3, 1986.

4) Kremp1, E., "An Experimenta1 Study of RoomTemperature Rate Sensitivity, Creep and Relaxation of Type 304 Stainless Steel," J. Mech. Phys. Solids, 27, 1979, 363-375.

5) Kujawski, D., Ka1lianpur, V., and Kremp1, E., "An Experimental Study of Uniaxial Creep, Cyclic Creep and Relaxation of AISI Type 304 Stainless Steel at Room Temperature," J. Mech. Phys. Solids, 28, 1980, 129-148.

6) Kremp1, E. and Lu, H., "The Hardening and Rate-Dependent Behavior of Fully Annealed AISI Type 304 Stainless Steel under Biaxial InPhase and Out-of-Phase Strain Cycling at Room Temperature," J. Engrg. Mat. and Tech., 106, $1984,376-382$

7) Kujawski, D. and Kremp1, E., "The Rate (Time) Dependent Behavior of Ti-7A-2Cb-1Ta Titanium Alloy at Room Temperature under Quasi-Static Monotonic and Cyclic Loading," J. Appl. Mech., 48, $1981,55-63$.

8) Kremp1, E. and Kallianpur, V.V., "Some Critical Uniaxial Experiments for Viscoplasticity at Room Temperature," J. Mech. Phys. Solids, 32, 1984, 301-314.

9) Kremp1, E., Lu, H. and Yao, D., "The Viscoplasticity Theory Based on Overstress Applied to the Modeling of a Nickel Base Superalloy at $815^{\circ} \mathrm{C}$," presented at the NASA Conf., Akron, $\mathrm{OH}$, June 1986, to appear in Proceedings.

10) Yao, D. and Kremp1, E., "Viscoplasticity Theory Based on Overstress. The Prediction of Monotonic and Cyclic Proportional and Nonproportional Loading Paths of an Aluminum Alloy," Int. J. Plasticity, $1,1985,259-274$.

11) Kremp1, E., McMahon, J.J. and Yao, D., "Viscoplasticity Based on Overstress with a Differential Growth Law for the Equilibrium Stress," Mech. of Materia1s, $\underline{5}, 1986,35-48$.
12) Phillips, A., "Combined Stress Experiments in Plasticity and Viscoplasticity. The Effects of Temperature and Time," in Plasticity of Metals at Finite Strain: Theory, Computation and Experiment, E.H. Lee and R.L. Mallett, eds., Stanford U., and Rensselaer Polytechnic Institute, 1982, 230.

13) Oytana, C., Delobelle, P. and Mermet, A., "Constitutive Equations Study in Biaxial Stress Experiments," J. Engrg. Mat. and Tech., 104, 1982, 1-11.

14) McDowe11, D., "A Two Surface Model for Transient Nonproportional Cyclic Plasticity: Part 1. Development of Appropriate Equations; Part 2. Comparison of Theory with Experiments, J. App1. Mechanics, 52, 1985, 298-308.

15) Kremp1, E., "Models of Viscoplasticity. Some Comments on Equilibrium (Back) Stress and Drag Stress," Report MML 87-2, Rensselaer Polytechnic, in print, Acta Mechanica.

16) Walker, K.P., "Research and Development Program for Nonlinear Structural Modeling with Advanced Time-Temperature Dependent Constitutive Relationships," NASA Report CR-165533, 1981 .

17) Cernocky, E.P. and Kremp1, E., "A Nonlinear Uniaxial Integral Constitutive Equation Incorporating Rate Effects, Creep, and Relaxation," Int. J. Nonlinear Mech., 14, 1979, 183-203.

18) Kremp1, E., "The Role of Servocontrolled Testing in the Development of the Theory of Viscoplasticity Based on Total Strain and Overstress," Amer. Soc. Testing and Mater., STP 765, $1982,5-28$.

19) Liu, M.C.M. and Kremp1, E., "A Uniaxial Viscoplastic Model Based on Total Strain and Overstress," J. Mech. Phys. Solids, 27, 1979, 377-391.

20) Kremp1, E., Lu, H., Satoh, M. and Yao, D. "Viscoplasticity Based on Overstress Applied to Creep-Fatigue Interaction," to appear in ASTM STP 942, American Society for Testing and Materials, 1987.

21) Ohashi, Y., Kawai, M. and Momose, T., "Effects of Prior Plasticity on Subsequent Creep of Type 316 Stainless Steel at Elevated Temperature," J. Engrg. Mat. and Tech., 108, 1986, 68-74. 
22) Krempl, E. and Lu, H., "The Path and Amplitude Dependence of Cyclic Hardening of Type 304 Stainless Steel at Room Temperature," to appear in Proceedings of 2nd Int. Conf. on Multiaxial Fatigue, Sheffield, England, 1985.

23) K emp1, E. and Yao, D., "The Viscoplasticity Th.eory Based on Overstress Applied to Ratchetting and Cyclic Hardening," to appear in Proceedings of 2nd Int. Conf. on Low-Cycle Fatigue and Elasto-Plastic Behavior of Materials, Munich, 1987.

24) Bena1la1, A. and Marquis, D., "Constitutive Equations Describing Nonproportional Effects

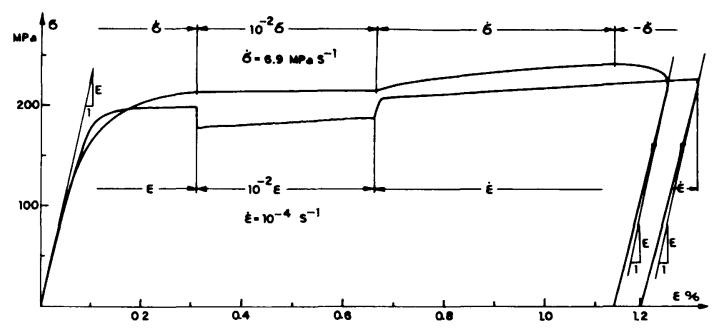

Fig. 1. The room temperature behavior of annealed type 304 stainless steel under stress or strain rate changes. Note that under strain control the effect of rate changes are much more pronounced than under stress control. Also observe the continued strain accumulation after reversal of stress rate in stress control. From [5].

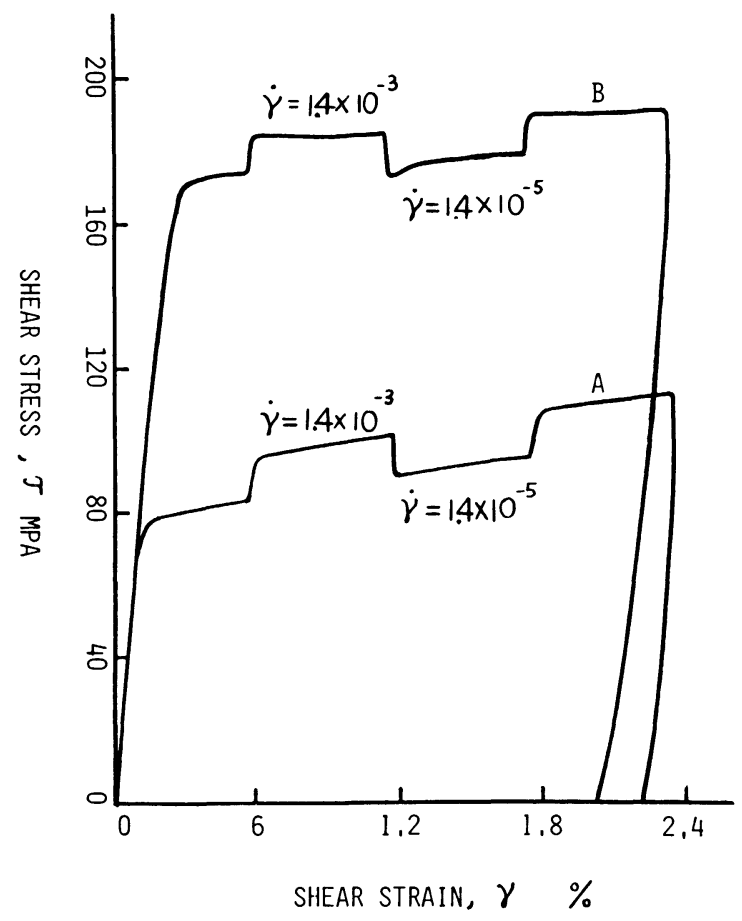

Fig. 2. The response of annealed (A) and precycled (B) type 304 stainless steel at room temperature to strain rate cycling. The stress level difference between the two strain rate curves is approximately the same for $A$ and $B$. in Cyclic Plasticity," in Constitutive Laws for Engineering Materials: Theory and Applica-

tions, eds. C.S. Desai et al., Elsevier Science Pub. Co., Inc., p.505, 1987.

25) Rohde, R.W., Jones, W.B. and Swearengen, J.C., "Deformation Modeling of Aluminum: Stress Relaxation, Transient Behavior and Search for Microstructura1 Correlations, Acta Metallurgica, 29, 1982, 41-52.

26) Swearengen, J.C. and Holbrook, J.H., "Internal Variable Models for Rate Dependent Plasticity: Analysis of Theory and Experiment," Res. Mechanica, 13, 1985, 92.

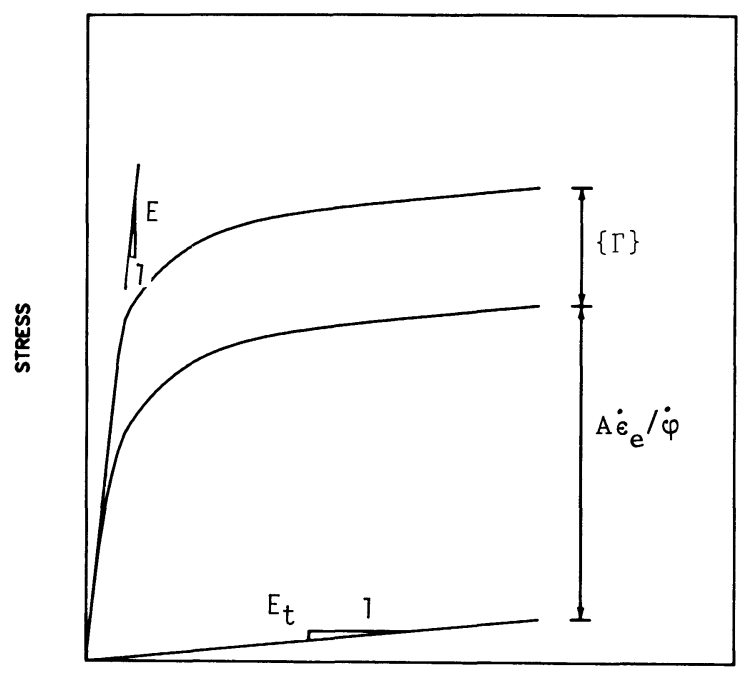

STRAN

Fig. 3, Schematic showing the properties of the asymptotic solution of the overstress model. $\{\Gamma\}$ is the viscous, $A \dot{\epsilon} e_{e} / \dot{\varphi}$ the rate-independent and $E_{t} \varepsilon_{e}$ the work-hardening contribution to the effective asymptotic stress $\left\{\sigma_{\mathbf{e}}\right\}$.

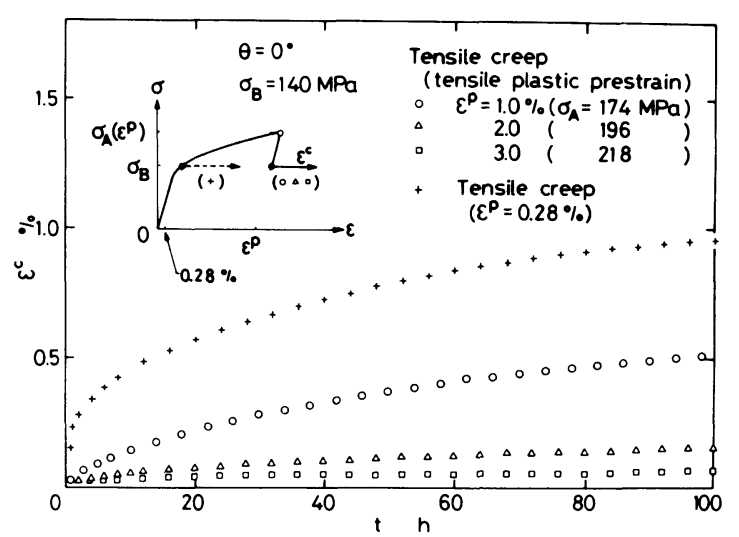

Fig, 4. The effect of prior plastic deformation on subsequent creep behavior. Stainless steel at $650^{\circ} \mathrm{C}$, Ohashi et a1. [21]. 


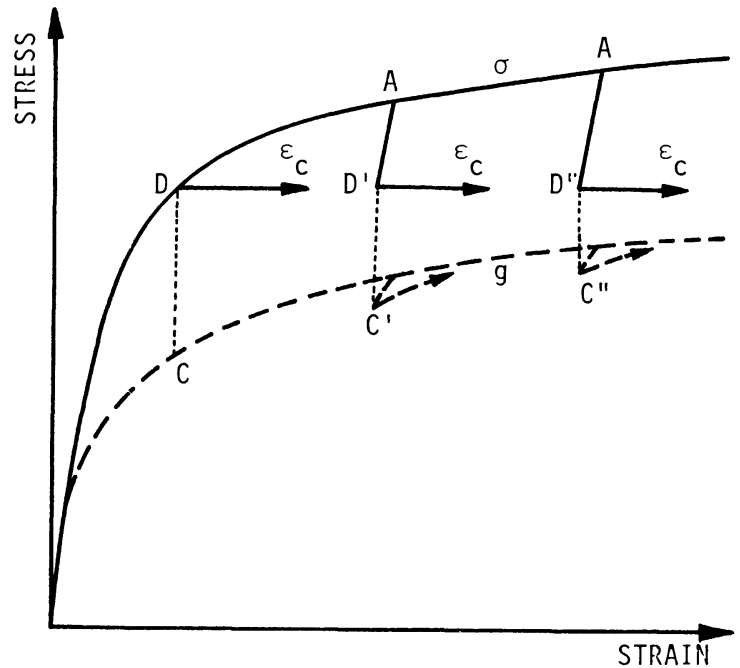

Fig. 5. Schematic to explain the results of Fig. 4. The growth of the equilibrium stress g (dashed curve) is such that the overstress at the beginning, of the creep tests is decreasing with increasing prior plastic strain, $D C>D^{\prime} C^{\prime}>D^{\prime \prime} C^{\prime \prime}$. Since the creep strain rate is an increasing function of the overstress, it decreases as well.

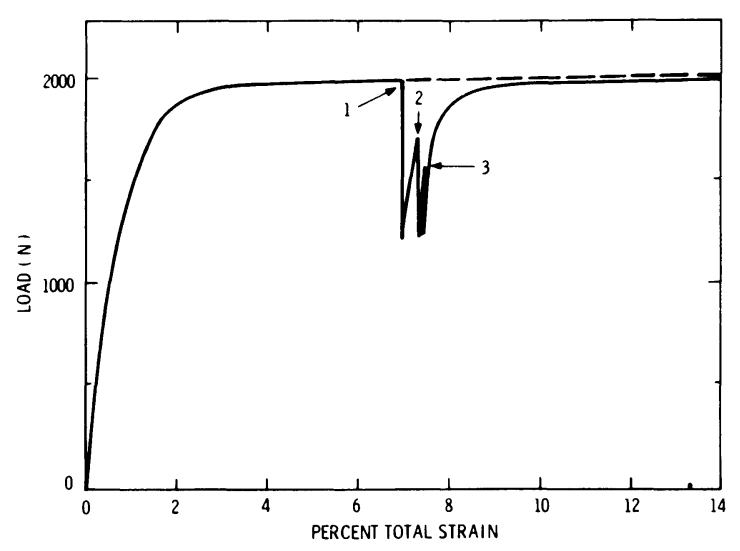

Fig. 6. Tracing of tensile stress-strain path of A1 sample showing initial relaxation and two subsequent relaxations after "elastic" reloading. Note that the extrapolation of the curve prior to relaxation lies above the curve generated upon reloading. From [26]; room temperature.

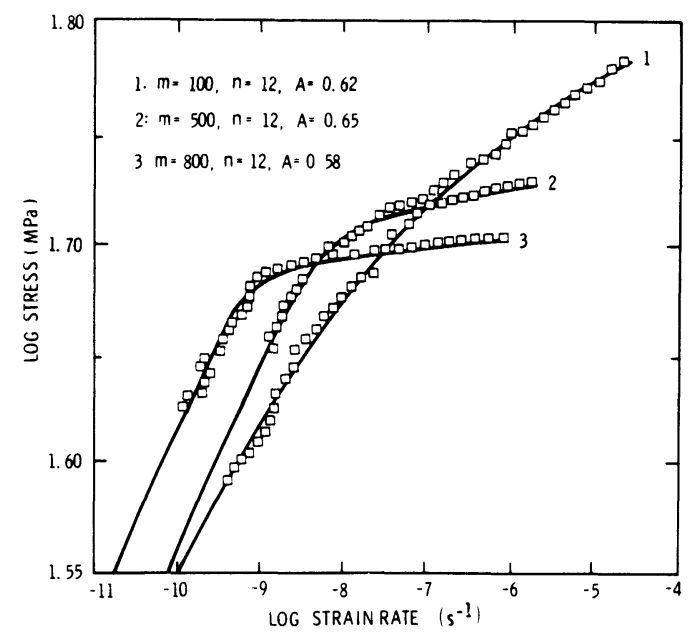

Fig. 7. Comparison of stress relaxation of A1 sample monotonically loaded in tension to $0.59 \%$ plastic strain (1); relaxed, reloaded an additional $0.013 \%$ plastic strain (2); relaxed, then reloaded an additional $0.005 \%$ plastic strain (3). Solid lines are model fit. From [26]; room temperature. 